\title{
Cabello bumano para valorar la exposición a arsénico en usuarios de agua contaminada de la comunidad La Fuente en, La Paz Centro, León.
}

M.Sc. Maximina Altamirano E.

Laboratorio de Contaminantes metálicos. Centro para la Investigación en Recursos Acuáticos de Nicaragua, UNAN-Managua maximina.altamirano@cira.unan.edu.ni

\section{M.Sc. Valeria Delgado}

Docente.

Centro para la Investigación en Recursos Acuáticos de Nicaragua, UNAN-Managua valeria.delgado@cira.unan.edu.ni

\author{
M.Sc. Silvia Fuentes Huelva \\ Área Analítica. \\ Centro para la Investigación en Recursos \\ Acuáticos de Nicaragua, UNAN-Managua \\ silvia.fuentes@cira.unan.edu.ni
}

Fecha de recepción: 20 de diciembre, 2019 / Fecha de aceptación: 03 de marzo, 2020

https://doi.org/10.5377/torreon.v9i25.9856

Palabras clave: Hidroarsenicismo, Biomarcador, Bioacumulación, Termalismo, Volcán Momotombo.

\section{RESUMEN}

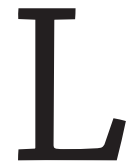

a presencia de Arsénico en las aguas superficiales y subterráneas de Nicaragua está relacionada con los geoambientes presentes en el país. El área de estudio se encuentra al NE del volcán Momotombo, localizado en el arco volcánico de la Depresión Nicaragüense, donde el material geológico predominante son rocas y sedimentos del Cuaternario aluvial y volcánico. La comunidad de La Fuente está conformada por 112 familias

Fe de erratas: Al presente artículo se agrega la siguiente nota al pie, que fue omitida involuntariamente en la primera versión publicada en junio del corriente año.

\section{Nota al pie}

Las autoras agradecen a los Fondos para Proyectos de Investigación FPI 2015-2017 de la UNAN-Managua por

94 medio del proyecto No. 19201504 por el apoyo económico otorgado para la ejecución de esta investigación. 
(1300 habitantes) que se abastecen de agua en su mayoría por fuentes artesanales (90 pozos excavados, y tres perforados, uno de ellos es comunitario). En un estudio previo se determinó Arsénico total en agua en un rango de 2.0 a $103.0 \mu \mathrm{g} / \mathrm{L}$, reportándose el máximo valor en un manantial termal; en donde un $70 \%$ de los sitios monitoreados (24 pozos) presentan valores no aptos para consumo (mayor o igual a $10 \mu \mathrm{g} / \mathrm{L}$ ). El Arsénico presente en el agua es absorbido por los tejidos, para posteriormente ser eliminado en la orina; pero cuando la ingesta es mayor que la excreción éste tiende a acumularse en cabello y uñas, al igual que en la orina y la sangre; siendo estos excelentes biomarcadores de exposición aguda y crónica a este metaloide. Considerando el tiempo de ingesta de agua contaminada con arsénico por pobladores de La Fuente, se decidió determinar si hay presencia de arsénico en los pobladores de La Fuente, utilizando el cabello humano como indicador biológico. Se identificó un grupo focal para este estudio considerando las mayores concentraciones de arsénico presentes en agua, así como también el mayor tiempo de ingesta, resultando un grupo poblacional de 43 mujeres y 9 varones. Se colectaron aproximadamente 5 gramos de cabello de cada individuo a ser evaluado, totalizando 52 muestras de cabello. Las concentraciones de arsénico detectadas en cabello oscilaron entre 0,014 y 0,925 $\mu \mathrm{g} \cdot \mathrm{g}^{-1}$; siendo menores a $1,00 \mu \mathrm{g} \cdot \mathrm{g}^{-1}$, valor establecido como criterio de toxicidad por el Centro Toxicológico de Quebec (CTQ). Las mayores concentraciones de arsénico están asociadas a la persona de mayor edad (mujer de 90 años) probablemente debido al mayor tiempo de exposición; y a la de menor edad (niño de 7 años) probablemente este contaminante se transmite de madre a hijos a través del cordón umbilical, presentando el neonato una concentración similar a su madre al momento de nacer. Los comunitarios fueron informados de sus resultados como parte del compromiso social con la población sujeta al estudio.

\section{INTRODUCCIÓN}

Son numerosas las poblaciones afectadas por la ingesta de arsénico en el agua, en todo el mundo, sin ser Nicaragua la excepción. Mundialmente, se considera un problema de salud pública que llega a tener diversos efectos carcinogénicos, neurotóxicos y mutagénicos (Rodríguez Barranco, M., Lacasaña, M., Aguilar Garduño, C., Alguacil , J., Gil, F., González Alzaga, B., et al. 2013). De acuerdo con la Agencia para sustancias tóxicas y el registro de enfermedades (ATSDR, 2011), la Agencia Internacional para la Investigación del Cáncer (IARC), el Programa Nacional de Toxicología y la Agencia para la Conservación del Ambiente (EPA) declararon al arsénico como factor de riesgo para la salud considerándolo como un carcinogénico humano conocido.

La Organización Mundial de la Salud estableció en el año 1993 el valor máximo admisible en agua de consumo humano la concentración de $50 \mu \mathrm{g} \cdot \mathrm{l}^{-1}$ para arsénico; sin embargo, debido a la toxicidad de este metaloide, este valor ha sido reducido mundialmente a $10 \mu \mathrm{g} \cdot \mathrm{l}^{-1}$ (WHO, 2011). 
El uso de biomarcadores para evaluar la toxicidad de agentes químicos presentes en el medio ambiente es cada día más difundido. Esta forma de evaluar el riesgo para las poblaciones expuestas, tiene desventajas como no considerar la variabilidad individual frente a un agente tóxico, la existencia de un largo período de latencia entre la exposición y el desarrollo de una enfermedad, por ejemplo, cáncer. En tanto que la utilización de datos moleculares hace que el período entre la exposición y la detección de marcadores de efectos genotóxicos sea más corto (Smith, Hopenhayn-Rich, Warner, Biggs, Moore, \& Smith, 1993).

Estudios exponen que el riesgo de cáncer bebiendo 1,6 litros de agua por día con 2,5 $\mu \mathrm{g} \cdot \mathrm{l}^{-1}$ es de 1/1000; y con niveles de $50 \mu \mathrm{g} \cdot \mathrm{l}^{-1}$ es de 21/1000; en donde los grupos poblacionales más vulnerables a la exposición de metales en el ambiente, son los infantes, ancianos y gestantes (Licata , Trombetta , Cristani , Naccari, Martino, \& Calo, 2005).

En Nicaragua son pocos los estudios que se han realizado evaluando la exposición humana al arsénico [ (Reyes Salgado \& Provedor Fonseca, 1998), (Gómez C., 2002), (Gómez Cuevas, 2007), (Genie Zepeda \& González Zambrana, 2017)], debido a la ingesta de agua y/o alimentos contaminados con este tóxico; o por contacto dérmico con suelos y rocas que naturalmente lo poseen (Wickre, Folt, Sturup, \& Karagas, 2004); así como también usando biomarcadores [(Wickre, Folt, Sturup, \& Karagas, 2004), (OPS/OMS, Nuevas Esperanzas, 2011)].

Los biomarcadores son parámetros que se cuantifican en un medio biológico procedente de orina, cabello, sangre y otros, que incluye la presencia de un contaminante en un medio biológico frente a la agresión de un xenobiótico a consecuencia de una exposición relativamente reciente (aguda o subaguda), a largo plazo (crónica) [(Gil \& Hernández, 2009), (Hernández, Gil, \& Tsatsakis, 2014)].

El cabello humano se ha usado como biomarcador para evaluar la exposición ambiental y ocupacional a ciertos tóxicos durante más de 50 años (Pereira, Ribeiro, \& Goncalves , 2004). (Chojnacka , Góreaka, \& Gorecki , 2006)] mencionan que esta preferencia del cabello sobre otros biomarcadores se asocia a:

- Que es una matriz estable.

- Que la muestra es de fácil obtención, transporte y almacenamiento.

- Que ha demostrado ser uno de los vehículos de excreción de metales, enormemente útil en la evaluación de intoxicaciones crónicas.

- Las concentraciones de arsénico encontradas en el cabello son 10 veces superiores a las existentes en la sangre y orina. 
- Que las concentraciones de arsénico en sangre y orina, reflejan una contaminación reciente; mientras que en el cabello es indicativo de exposición crónica.

A la vez, (Repetto, 1995) menciona que el cabello humano revela el consumo de aguas contaminadas con arsénico inorgánico, así como el consumo de alimento contaminado con carácter retrospectivo; por lo que el cabello es un verdadero calendario del uso y consumo de aguas contaminadas, drogas y alimentos.

La concentración de arsénico en el cabello depende del tiempo de exposición y la velocidad de crecimiento de este; mientras más largo sea, representa mayor tiempo de exposición. El crecimiento varía entre 0.75 y $1.35 \mathrm{~cm}$ por mes, aunque variables como la edad, el género y el embarazo pueden alterar de manera significativa esta velocidad (Harker, 1993).

La ATSDR considera que valores mayores de 1,00 mg/kg de arsénico total en cabello humano son nocivos para la salud por sus efectos tóxicos y cancerígenos (ATSDR, 2011); de igual manera, el Centro Toxicológico de Quebec (CTQ) establece el mismo criterio de toxicidad (CTQ, 1980).

Fue hasta el 2004 que se realizó el primer estudio en Nicaragua utilizando biomarcadores. En esta investigación, se determinó el grado de exposición al arsénico y mercurio de los niños y adultos de la comunidad minera en Siuna, utilizando uñas como biomarcador (Wickre, Folt, Sturup, \& Karagas, 2004). Siete años después se realizó el segundo estudio utilizando biomarcadores, en este caso, cabello humano; con el fin de determinar el impacto del consumo de agua contaminada con arsénico en la salud de la población de cinco comunidades del municipio de Telica (OPS/OMS, Nuevas Esperanzas, 2011).

\section{1. Área de Estudio}

En el Municipio de La Paz Centro, se encuentra localizada la comunidad rural La Fuente, con una población de 1300 habitantes distribuidos en 120 familias. El área de estudio tiene una extensión aproximada de $15 \mathrm{~km}^{2}$ (Figura 1). Se ubica al Noreste de las faldas septentrionales del complejo volcánico Momotombo-El Hoyo y del edificio de la caldera Monte Galán (OLADE, 1982). 


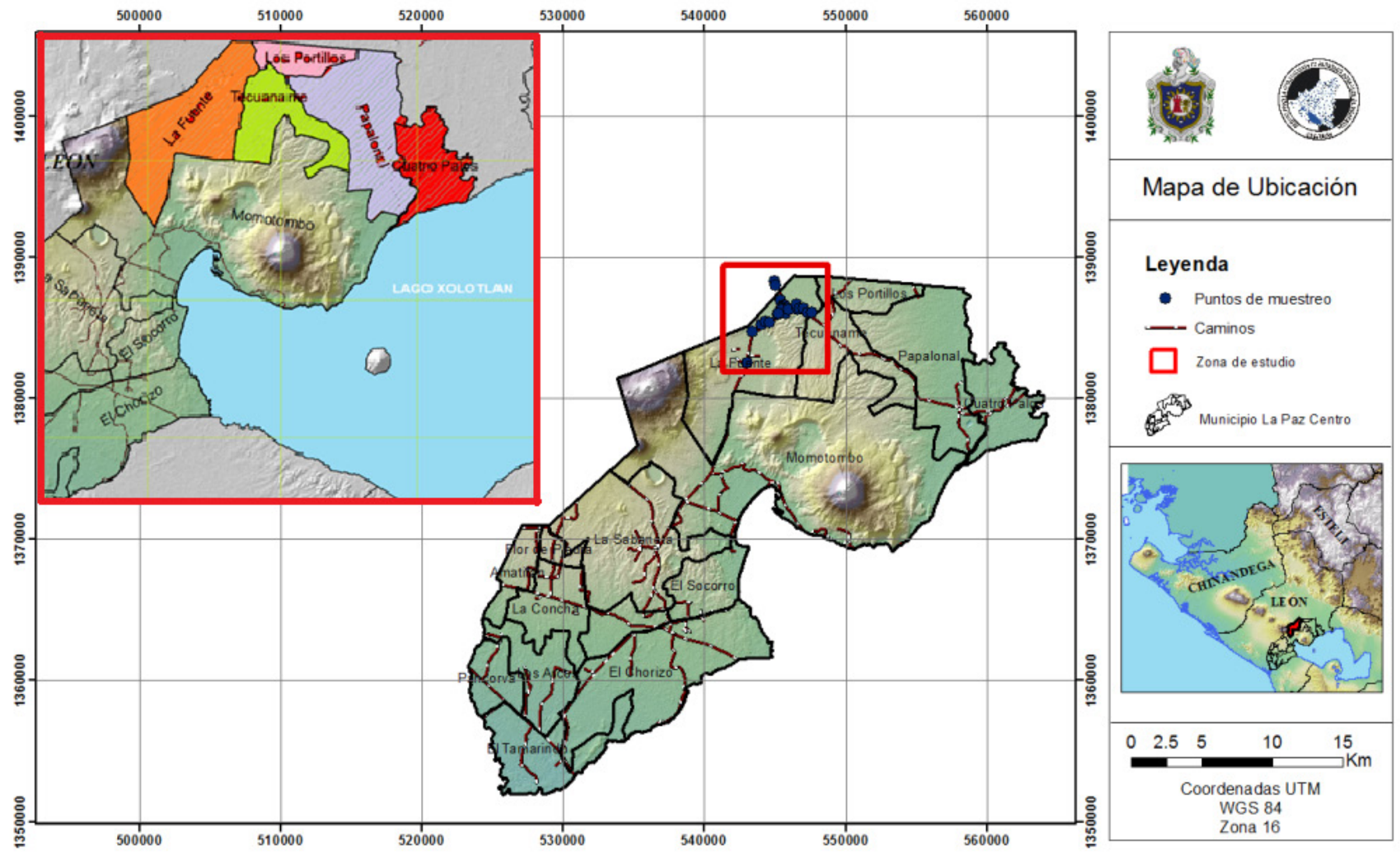

Figura 1. Macro y micro localización de la Comunidad Rural La Fuente, La Paz Centro. Tomada de (Altamirano., 2017 in proceso)

\section{METODOLOGÍA}

\subsection{Materiales y métodos}

El presente estudio es resultado de una investigación previa realizada con Fondos de Proyectos para la Investigación de la UNAN-Managua (FPI \#12201504), (Altamirano E, 2017); llevado a cabo en la misma comunidad, en donde se determinaron las concentraciones de arsénico en agua de 34 pozos de los 93 existentes; resultando un rango de concentración de 2 a $103 \mu \mathrm{g} \cdot \mathrm{l}^{-1}$, que corresponde a que un $70 \%$ de los pozos tengan niveles de arsénico por arriba de los $10 \mu \mathrm{g} \cdot \mathrm{l}^{-1}$ establecidos por la OMS para el consumo humano (OMS, 2011). Esta información fue utilizada como línea base para la ejecución de este trabajo de investigación.

El primer componente de esta etapa, consistió en realizar entrevistas individuales a los pobladores de La Fuente, enfocándose en las diferentes patologías que padecen los habitantes de esta comunidad.

Se realizó una conversación informal con los líderes comunitarios, en donde las principales manifestaciones expresadas durante estos diálogos son:

1. Enfermedades renales. 
2. Hipertensión, diabetes y cáncer; estos tres padecimientos se presentan en menor escala que las enfermedades renales; y afectan más a las mujeres que a los varones.

3. Dermatitis: los pobladores expresan padecimientos de alergias y lesiones verrucosas en la piel, las cuales asocian con problemas de calidad del agua.

Una vez obtenida la información de los comunitarios, se procedió a realizar la selección del grupo poblacional que sería sometido al análisis de arsénico en cabello. Para esto, se consideró:

1. El contenido de arsénico en el agua de consumo.

2. El tiempo de consumo de esa agua (tiempo de exposición).

3. La edad y género de la persona.

En el procedimiento para la toma de muestra de cabello (Figura 2), se debe considerar:

- Que ésta sea fácil de colectarse.

- Que se cumpla con el protocolo de muestreo:

a. Antes de proceder a la toma de muestra, se lee el consentimiento informado si es un adulto; si son niños se solicita el permiso a los padres del menor para la toma de la muestra.

b. Cortar el cabello cercano a la parte inferior del cuello (en la naciente del cabello), ya que éste no debe estar expuesto al sol, al viento, al polvo (Figuras 2a, 2b, 2c y 2d). En este procedimiento se usan guantes y tijeras esterilizadas.

c. Captar aproximadamente 5 gramos de cabello, y almacenarlo de manera individual en bolsas ziploc; las cuales serán rotuladas con los datos de cada individuo monitoreado:

d. Fecha y hora de muestreo,

e. Nombre de la persona muestreada.

f. Sexo, edad y tiempo de vivir en la comunidad.

g. Las muestras no requieren ser preservadas.

h. Finalmente, las muestras de cabello fueron embaladas en contenedores apropiados.

Se siguió el procedimiento operativo para colecta de muestra (PON- CM-06) establecido en el Laboratorio de Contaminantes Metálicos del CIRA/UNAN-Managua. 

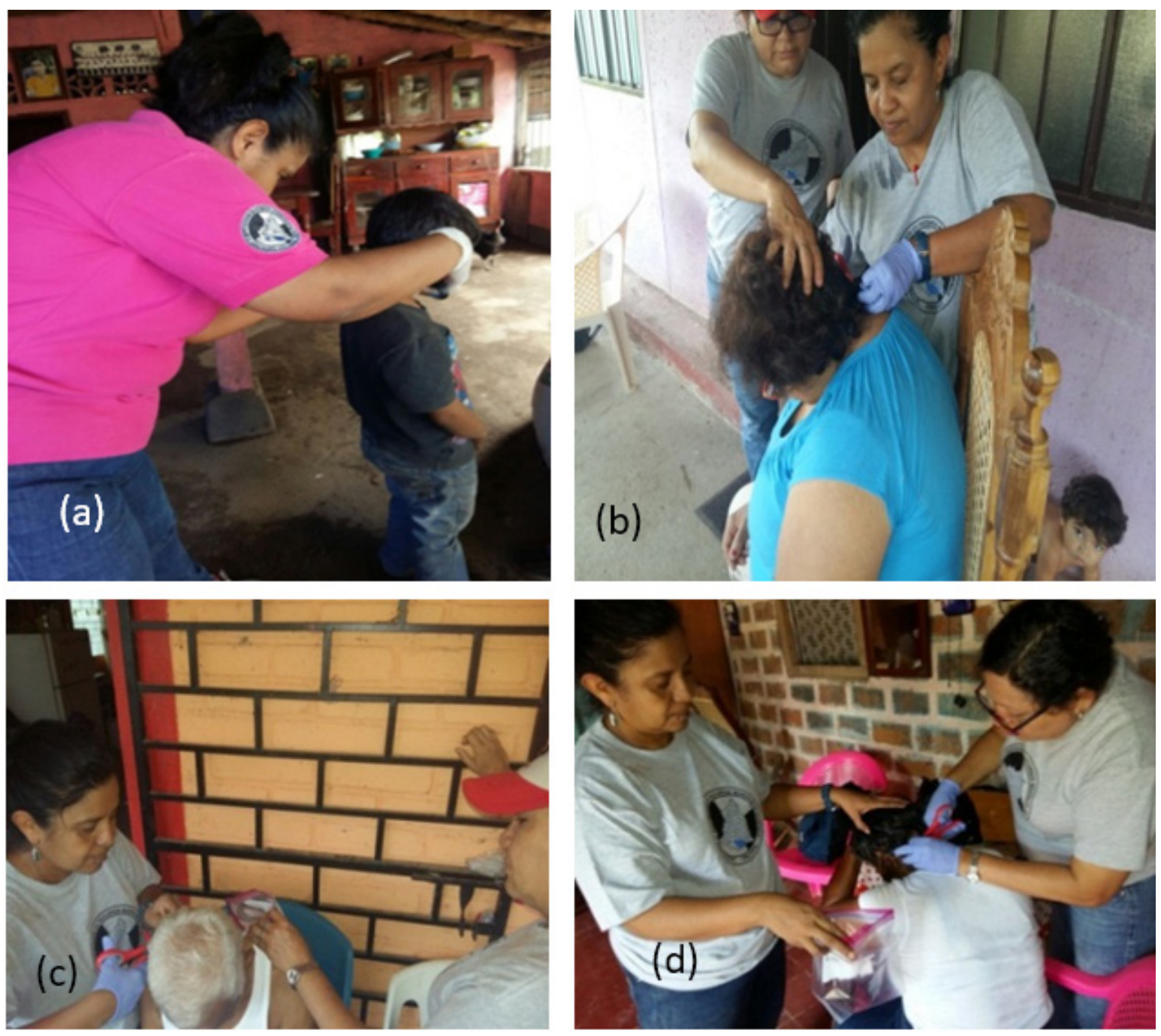

Figura 2 ( $a, b, c y d)$. Toma de muestra de cabello en el grupo poblacional de La Fuente.

\section{RESULTADOS}

Para la interpretación de los resultados, únicamente se usarán las iniciales del grupo poblacional muestreado para este fin; esto con el objetivo de respetar su identidad personal y su integridad física; garantizando de esta manera el compromiso de confidencialidad expresado en el consentimiento informado.

En la Tabla 1 se reportan las concentraciones de arsénico en cabello humano de la muestra poblacional de La Fuente; con un rango de concentraciones que osciló de 0.011 a $0.925 \mu \mathrm{g} \cdot \mathrm{g}^{-1}$. El rango etario evaluado fue de 7 a 90 años, con un valor de ingesta de arsénico de 2 a $103 \mu \mathrm{g} \cdot \mathrm{l}^{-1}$; y un rango de consumo de agua contaminada con arsénico de 7 meses a 90 años. 
Tabla 1. Concentraciones de arsénico en cabello humano $\left(\mu g \cdot g^{-1}\right)$ del grupo poblacional evaluado en La Fuente.

\begin{tabular}{|c|c|c|c|c|c|c|c|c|c|}
\hline Usuario & $\begin{array}{c}\text { Arsénico } \\
\left(\mu \mathrm{g} \cdot \mathrm{g}^{-1}\right)\end{array}$ & $\begin{array}{l}\text { Edad } \\
\text { (años) }\end{array}$ & Sexo & $\begin{array}{c}\text { Rango } \\
\text { de } \\
\text { consumo } \\
\text { (años) }\end{array}$ & Usuario & $\begin{array}{c}\text { Arsénico } \\
\left(\boldsymbol{\mu g} \cdot \mathbf{g}^{-1}\right)\end{array}$ & $\begin{array}{c}\text { Edad } \\
\text { (años) }\end{array}$ & Sexo & $\begin{array}{c}\text { Rango } \\
\text { de } \\
\text { consumo } \\
\text { (años) }\end{array}$ \\
\hline REC & 0,464 & 49 & F & 30 & YH & 0,186 & 61 & F & 61 \\
\hline NEA & 0,640 & 30 & $\mathrm{~F}$ & 30 & FCR & 0,156 & 26 & F & 26 \\
\hline MDL & 0,011 & 27 & F & 8 & $\mathrm{LC}$ & 0,266 & 9 & F & 9 \\
\hline MPV & 0,011 & 40 & M & 7 meses & PRM & 0,106 & 30 & F & 30 \\
\hline $\mathrm{MBC}$ & 0,011 & 40 & F & 7 meses & ERH & 0,076 & 50 & F & 50 \\
\hline AEP & 0,011 & 82 & $\mathrm{~F}$ & 70 & CA & 0,114 & 60 & M & 60 \\
\hline GG & 0,054 & 58 & F & 40 & $\mathrm{AA}$ & 0,227 & 20 & M & 20 \\
\hline ARC & 0,011 & 79 & F & 75 & MMR & 0,029 & 14 & F & 14 \\
\hline MAM & 0,138 & 22 & F & 20 & $\mathrm{ADV}$ & 0,194 & 5 & F & 5 \\
\hline FRO & 0,073 & 70 & $\mathrm{~F}$ & 33 & KVA & 0,162 & 6 & F & 6 \\
\hline JAE & 0,011 & 89 & $\mathrm{~F}$ & 88 & DVR & 0,199 & 5 & F & 5 \\
\hline RJA & 0,029 & 64 & M & 45 & $\mathrm{NCH}$ & 0,124 & 50 & M & 50 \\
\hline ANH & 0,154 & 42 & $\mathrm{~F}$ & 38 & EPR & 0,416 & 60 & F & 60 \\
\hline JRR & 0,209 & 70 & $\mathrm{~F}$ & 68 & SA & 0,281 & 8 & F & 8 \\
\hline LA & 0,040 & 69 & $\mathrm{~F}$ & 66 & DPA & 0,120 & 5 & F & 5 \\
\hline LSH & 0,014 & 54 & F & 20 & $\mathrm{OP}$ & 0,057 & 12 & $\mathrm{M}$ & 12 \\
\hline NSD & 0,194 & 50 & $\mathrm{~F}$ & 20 & SEP & 0,393 & 54 & F & 54 \\
\hline MIRR & 0,167 & 56 & F & 42 & JLE & 0,493 & 6 & F & 6 \\
\hline MRV & 0,215 & 80 & $\mathrm{~F}$ & 20 & JEP & 0,248 & 52 & F & 52 \\
\hline FRO & 0,011 & 60 & F & 20 & KLM & 0,133 & 23 & F & 23 \\
\hline MHS & 0,011 & 76 & F & 52 & LEM & 0,158 & 18 & F & 㞧 \\
\hline EV & 0,044 & 72 & F & 67 & ASEP & 0,129 & 23 & F & 气ุ \\
\hline JVM & 0,644 & 80 & F & 80 & MLM & 0,194 & 24 & F & $\tilde{n}$ \\
\hline DR & 0,314 & 73 & $\mathrm{~F}$ & 72 & ARS & 0,254 & 25 & $\mathrm{M}$ & 夏 \\
\hline MSG & 0,125 & 43 & F & 43 & AYJ & 0,319 & 18 & M & 끙 \\
\hline EIM & 0,925 & 90 & F & 90 & AVR & 0,824 & 7 & $\mathrm{M}$ & $\frac{n 5}{101}$ \\
\hline
\end{tabular}


De los 52 habitantes a los que se les analizó arsénico total en cabello humano, 44 (84.62\%) reportan valores de arsénico por arriba del límite de detección; desde 0,014 a 0,925 $\mu \mathrm{g} \cdot \mathrm{g}^{-1}$. Las mayores concentraciones de arsénico fueron encontradas en cuatro usuarios: EIM con 90 años $\left(0,925 \mu \mathrm{g} \cdot \mathrm{g}^{-1}\right)$; AVR con 7 años $\left(0,824 \mu \mathrm{g} \cdot \mathrm{g}^{-1}\right)$; JVM con 83 años $\left(0,644 \mu \mathrm{g} \cdot \mathrm{g}^{-1}\right)$; y NEA con 30 años $\left(0,640 \mu \mathrm{g} \cdot \mathrm{g}^{-1}\right)$ quienes viven en esta comunidad desde su nacimiento (Tabla 1; Figura 3). Ninguno de ellos se encuentra aún expuesto a concentraciones consideradas como tóxicas en las personas [criterio establecido de $1 \mu \mathrm{g} \cdot \mathrm{g}^{-1}$ (CTQ, 1980)]. Sin embargo, el poblador EIM quien reporta la mayor concentración detectada en este estudio, se encuentra muy cerca del criterio de toxicidad.

Cabe mencionar que ocho personas reportaron concentraciones de arsénico en su cabello menores que el límite de detección del método (Tabla 1). De estas: AEP, ARC y JAE ingieren agua Fuente Pura, por lo que no están expuestos a la ingesta de aguas de la comunidad; FRO y MHS nacieron en la comunidad de Tecuaname, donde este tóxico está ausente y tienen poco tiempo de residir en La Fuente; MBC vive prácticamente en Momotombo y visita La Fuente una vez al mes, por lo que ocasionalmente ha ingerido agua con una concentración de 50,90 $\mu \mathrm{g} \cdot \mathrm{l}^{-1}$. Finalmente, MDL y MPV tienen siete meses de residir en la comunidad.

$\mathrm{Al}$ analizar las particularidades de EIM, se observa que es la persona de mayor edad en el grupo poblacional, que tiene mayor tiempo de prevalencia en la comunidad, por lo que se considera que desde su nacimiento ha consumido agua con una concentración de arsénico de $12,75 \mu \mathrm{g} \cdot \mathrm{l}^{-1}$. AVR es quien reporta el segundo valor más alto de arsénico en cabello $\left(0,824 \mu \mathrm{g} \cdot \mathrm{g}^{-1}\right)$, y a la vez, se encuentra dentro del grupo más joven de los evaluados. Desde su nacimiento ha consumido agua con $23.27 \mu \mathrm{g} \cdot \mathrm{l}^{-1}$ de arsénico. JVM y NEA tienen concentraciones de arsénico en cabello similares $\left(0,644 \mu \mathrm{g} \cdot \mathrm{g}^{-1}\right.$ y $0,640 \mu \mathrm{g} \cdot \mathrm{g}^{-1}$, respectivamente); no obstante, hay una gran diferencia de edad entre ambos habitantes: JVM tiene 83 años, mientras que NEA tiene 30 años. Aunque ambos (JVM y NEA) han ingerido agua contaminada con arsénico desde su nacimiento, las concentraciones de arsénico en las fuentes de agua que consumieron difieren de $14,8 \mu \mathrm{g} \cdot 1^{-1} \mathrm{a}$ $34,84 \mu \mathrm{g} \cdot \cdot^{-1}$, respectivamente.

Como puede apreciarse, a mayor concentración de arsénico en el agua de ingesta, mayor es la exposición de la persona, aunque el tiempo de ingesta de agua contaminada con este metaloide sea menor; esto se refleja en una mayor concentración de arsénico detectada en cabello. 


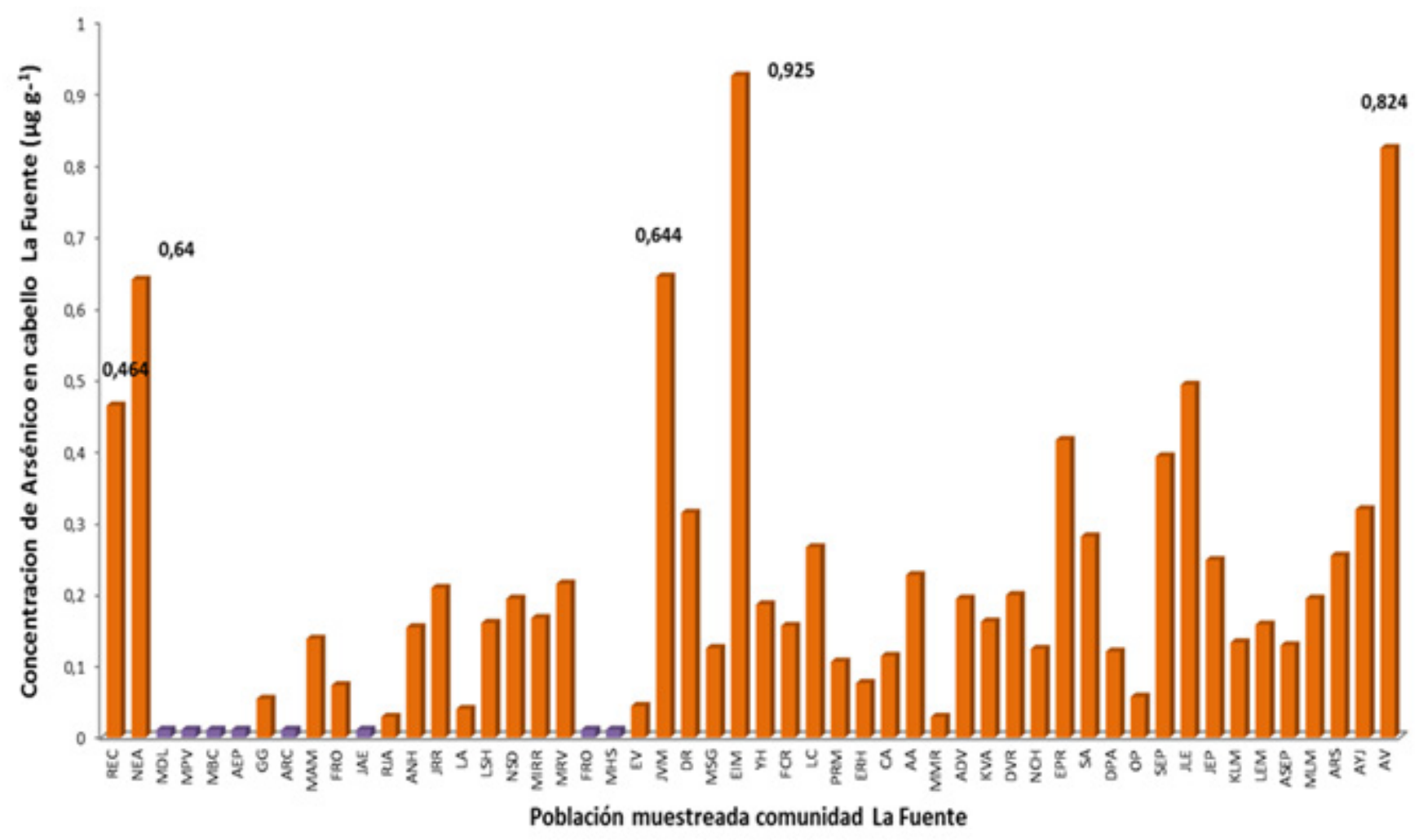

Figura 3. Concentraciones de arsénico (As) en cabello humano $\left(\mu g \cdot g^{-1}\right)$ en la comunidad de La Fuente.

Un hallazgo en este estudio, es el caso de dos niñas gemelas (ADVR y DVR) de 7 años, las cuales fueron evaluadas, reportando concentraciones de arsénico en cabello similares: 0,194 y 0,199 $\mu \mathrm{g} \cdot \mathrm{g}^{-1}$, respectivamente; ambas han ingerido desde su nacimiento agua con una concentración de arsénico de 23,52 $\mu \mathrm{g} \cdot \mathrm{l}^{-1}$ (PE-IR). A su corta edad, se percibe la bioacumulación de este tóxico en el organismo; distribuyéndose uniformemente por todos los órganos y tejidos excepto cabello y uñas, en donde se ha observado un tiempo de retención más largo, aunque después de la exposición puede concentrarse inicialmente más en el hígado, riñón, y en los pulmones (Bertolero, Marafante, Edel Rade , \& Pietra , 1981). Por último, en este caso en particular, queda evidenciado una estrecha relación entre edad-tiempo de ingesta-concentración de arsénico en el agua de consumo.

En la comunidad vecina de El Papalonal, AB de 103 años reportó una concentración de arsénico en cabello $<0,011 \mu \mathrm{g} \cdot \mathrm{g}^{-1}$, consumiendo agua con $<0,99 \mu \mathrm{g} \cdot \mathrm{l}^{-1}$ de arsénico desde su nacimiento [(Herrera, En ejecución), (Stynze Ramírez, En Ejecución)]. Al comparar estas concentraciones de arsénico en cabello con las concentraciones de EIM (0,925 $\mu \mathrm{g} \cdot \mathrm{g}^{-1} ; 90$ años; consumiendo agua con $12,75 \mu \mathrm{g} \cdot \mathrm{l}^{-1}$ desde su nacimiento), se observa claramente que el uso de este biomarcador (cabello) provee un registro a largo plazo sobre la exposición al arsénico por ingesta de agua con altas concentraciones de este carcinogénico (Chowdhury, et al., 2000). 
De manera general, los resultados muestran que las mayores concentraciones de arsénico en cabello se dan en niños y ancianos. (Licata , Trombetta , Cristani , Naccari , Martino, \& Calo, 2005) mencionan que esto es debido a que los niños tienen menor peso que los adultos y son más activos, lo que los conlleva a tomar más agua generando mayor ingesta y exposición; lo mismo ocurre con los ancianos, estos ingieren menos alimentos provocando cierta desnutrición; y son menos activos, favoreciendo la mayor acumulación de este carcinógeno. Por todo lo expuesto, el análisis de cabello es una herramienta tal vez única por su característica especial de extrapolar resultados en el tiempo siendo de gran importancia para ser usada en toxicología pre y postmortem, como fue el caso de Napoleón Bonaparte.

Se determinó que el 15,4 \% de los usuarios evaluados (Figura 4) no tienen arsénico en su organismo, ya que reportan valores menores que el límite de detección del método $\left(0,011 \mu \mathrm{g} \cdot \mathrm{g}^{-1}\right)$; por lo que son considerados sin exposición a este tóxico. En un segundo grupo, donde se localiza la mayoría de la población evaluada y quienes reportan concentraciones de arsénico de 0,012 a $0,499 \mu \mathrm{g} \cdot \mathrm{g}^{-1}$ en su sistema, totalizan un $77 \%$ de personas expuestas a este contaminante en su ingesta diaria (Figura 4). Finalmente, existen dos sub-grupos poblacionales correspondiendo cada uno de ellos a un 3,84\% de la población total evaluada; pero cuyo rango total de concentración de arsénico detectado en el cabello se encuentran entre 0,5 a 0,925 $\mu \mathrm{g} \cdot \mathrm{g}^{-1}$, siendo estas las personas más expuestas y con mayor riesgo en su salud (Figura 4). Estos resultados muestran una media de $0,1550 \mu \mathrm{g} \cdot \mathrm{g}^{-1}$, con una moda de $0,011 \mu \mathrm{g} \cdot \mathrm{g}^{-1}$, para un promedio de $0,1998 \mu \mathrm{g} \cdot \mathrm{g}^{-1}$; con un mínimo de 0,011 y el máximo 0,925 $\mu^{g^{-1}}$ de arsénico en cabello humano.

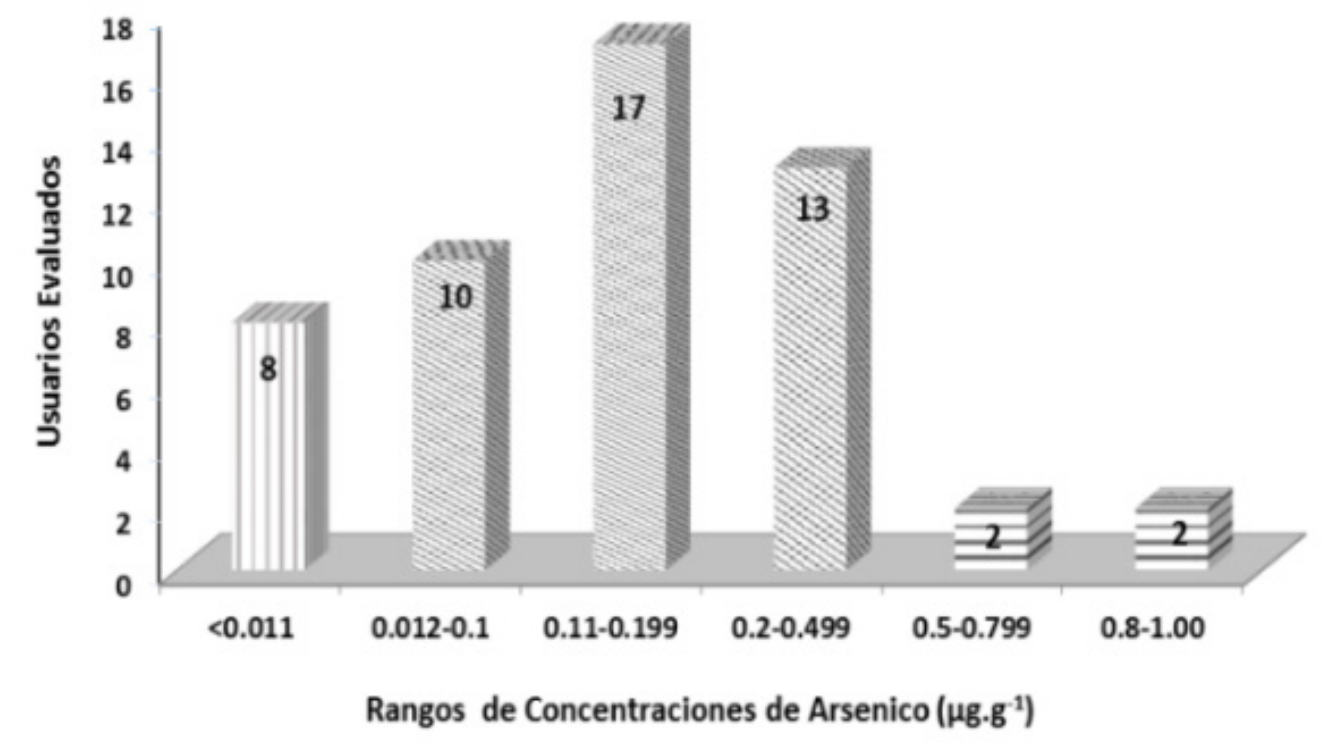

Figura 4. Rangos de concentraciones de arsénico total en cabello humano $\left(\mu g \cdot g^{-1}\right)$ en usuarios de fuentes de aguas contaminadas comunidad rural La Fuente

Como parte del componente social de esta investigación y del compromiso adquirido con los comunitarios de La Fuente, se realizó una presentación el 25 de mayo del año 2017 en la Escuela 
Santa Ana de esta comunidad. A esta presentación, se invitaron a: 1) la comunidad en general de La Fuente; 2) a las autoridades locales de los CAPs de El Terrero, Tecuaname, El Papalonal y La Fuente; 3) al Cro. César Torres Salgado, encargado de la Gestión de Riesgo de la Alcaldía de La Paz Centro; 4) a los miembros del grupo Samaritan's Purse Canada, quienes instalaron filtros para la remoción de bacterias en esta comunidad; 5) a la directora de la Escuela Santa Ana; y 6) personal del CIRA/UNAN-Managua (a la Jefa del Área de Investigación y Desarrollo, a la encargada de la Función de Proyección y Extensión Universitaria, y al personal del Laboratorio de Contaminantes Metálicos). En total, se contó con la participación de aproximadamente 100 comunitarios, entre adultos y niños. La presentación estuvo a cargo de la MSc. Maximina Altamirano, ejecutora de este proyecto y Jefa del Laboratorio de Contaminantes Metálicos del CIRA/UNAN-Managua.

Después de la presentación de los resultados y hallazgos reportados en este estudio, los pobladores se mostraron interesados en que se les explicara el porqué de esos valores, que para algunos de ellos resultaron preocupantes; ya que como manifestaron, ellos no consideraban que su agua estuviera contaminada, ya que física y organolépticamente no se observa nada anormal; y mucho menos que ellos fuesen portadores de este contaminante. Los pobladores consultaron qué medidas debían de tomar para garantizar la ingesta de agua segura, a la vez que manifestaron estar dispuestos a realizar los cambios que sean necesarios para evitar seguir consumiendo ese tóxico que afecta a su salud. El personal del CIRA/UNAN-Managua, orientó hacer uso de las fuentes de agua que presentan valores menores a los $10 \mu \mathrm{g} \cdot \mathrm{l}^{-1}$ de arsénico, las cuales, de acuerdo a la OMS, son las recomendadas para consumo humano. Finalmente se realizó la entrega de los resultados en copia física a cada uno de los usuarios evaluados (Figura 5). 

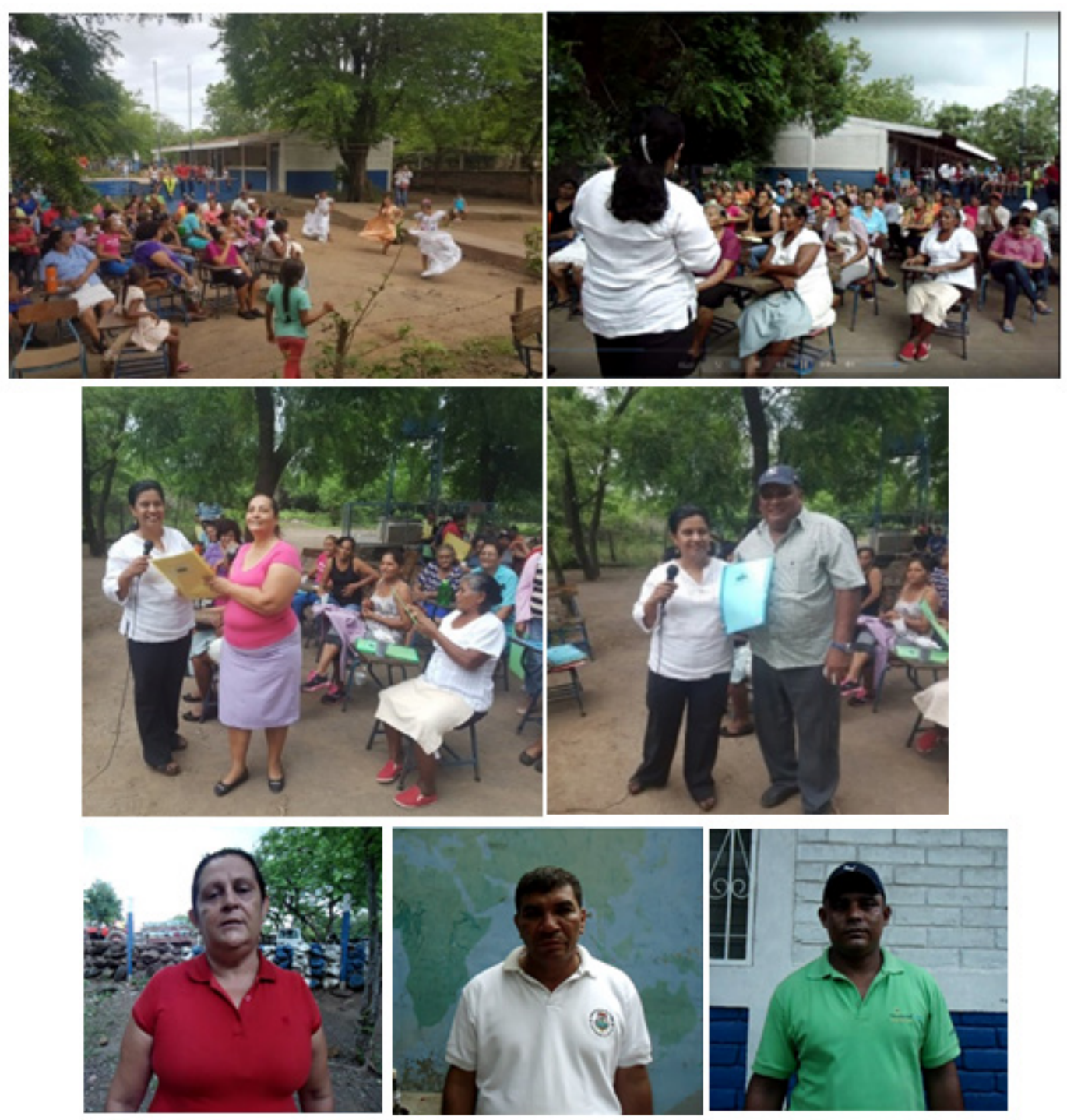

Figura 5. Presentación y entrega de los resultados de arsénico (As) en cabello humano al grupo poblacional evaluado de la comunidad de La Fuente.

\section{CONCLUSIONES}

1. Debido a las características geológicas delárea de estudio, el arsénico está presente de manera natural, contaminando las aguas superficiales y subterráneas; generando un problema de salud pública debido a la ingesta de agua con elevadas concentraciones de este genotóxico.

2. La evaluación a la exposición de arsénico usando cabello humano como biomarcador, indicó que las personas evaluadas no superan los valores de toxicidad establecidos por el CTQ; aún en periodos prolongados de ingesta. 
3. En la comunidad de La Fuente el factor preponderante en la prevalencia de las concentraciones de Arsénico total en cabello humano, es el contenido arsenical de sus fuentes de aguas subterráneas de origen geogénico, donde el $70 \%$ de las fuentes exceden la normativa asumida por el país.

4. Los resultados indican las condiciones actuales sobre la presencia de arsénico total en las muestras de cabello a los usuarios evaluados y el riesgo de exposición al seguir consumiendo agua contaminada con este metaloide.

5. Dada la prevalencia de este tóxico en la población con mayor tiempo y concentración de exposición al arsénico, se orientaron las fuentes de abastecimiento de agua segura para consumo humano.

\section{REFERENCIAS}

Altamirano E, M. (2017). Contaminación natural por arsénico en las aguas subterráneas de la comunidad rural "La Fuente", para sugerir y promover el uso de fuentes alternativas de agua segura municipio la Paz Centro, León. Managua, Nicaragua.

ATSDR. (2011). The priority list of hazardous Substances, Department of Health and Human Services, Public health Services. Agency for toxic Substance and Disease Registry.

Bertolero, F., Marafante, E., Edel Rade , J., \& Pietra , R. (1981). Biotransformation and intracelular binding of arsenic in tissues of rabbits after intraperitioneal administration of 74 As labiled arsenite toxicology. 20,35-44.

Chojnacka , K., Góreaka, H., \& Gorecki , H. (2006). The efect of age, sex , smoking habit and hair color on the composition of hair . Environ Toxicol pharmacol, 22.
Chowdhury, U. K., Biswas, B. K., Roychowdhury, T., Samanta, G., Mandal, B. K., Basu, G. K., et al. (2000). Groundwater arsenic contamination in Bangladesh and West Bengal, India. Environ. Health Perspect., 108(5), 393396.

CTQ. (1980). Méthode mormalisé pour le dosage de l'arsenic dans les cheveux par absorption atomique avec four de grafite M-126-A. Centre de Toxicologie du Quebec.

Genie Zepeda, E., \& González Zambrana, R. (2017). Efectos a la salud por exposición crónica a arsénico en agua de bebida en habitantes adultos de comunidades rurales del Municipio Larreynaga-Malpaisillo. Tesis de grado, Managua.

Gil , F., \& Hernández, A. F. (2009). Significance of biochemical markers in applied toxicology. (B. Ballantyne, T. C. Marrs , \& T. Syversen, Edits.) In general and Applied Toxicology, 2, 847 - 858. 
Gómez C., A. (2002). Monitoreo y Atención de intoxicados con Arsénico en El Zapote, Municipio de San Isidro, Departamento de Matagalpa, Nicaragua 1994-2002. Managua: UNICEF.

Gómez Cuevas, A. (2007). Efectos Cutáneos y Respiratorios en personas con intoxicación crónica por arsénico en agua El Zapote, Municipio de San Isidro - Matagalpa, Nicaragua. 2007. Tesis de Maestría, Managua.

Harker, M. R. (1993). Anatomy and Phisiology of hair. Forensic Science. International, 63.

Herrera, D. (En ejecución). Valoración de la exposición a arsénico mediante el uso del biomarcador cabello humano en los usuarios de fuentes de agua contaminada en las comunidades: Tecuaname y el Papalonal del municipio de La Paz Centro. Tesis de Maestría, Managua.

INAA. (2001). Normas Técnicas para el diseño de abastecimiento y potabilización de agua. Normas NTOM 09003-99. Managua.

Licata , P., Trombetta , D., Cristani , M., Naccari , C., Martino , D., \& Calo , M. (2005). Heavy metals in liver and muscle of bluefin tuna (Thunnus thynnus) caught in the Straits of Lillo,

OMS. (2011). Guías para la calidad del agua de consumo humano, Cuarta edición que incorpora la primera ADENDA.

OPS/OMS, Nuevas Esperanzas. (2011). Estudio de la Contaminación del Agua por
Arsénico en 1853 el Municipio de Télica, León. Télica, León, Nicaragua.

Pereira, R., Ribeiro , R., \& Goncalves , F. (2004). Scalp hair analysis as a tool in assessing human exposure to heavymetals (S Domingos mine, Portugal ). SCI total Environ.

PON. (2010). Procedimiento Operativo Normalizado (PON) para la determinación de arsénico total en cabello humano como un biomarcador de exposicion por absorcion atomica - generación de hidruro. Managua: CIRA/UNAN-Managua.

Repetto, M. (1995). Toxicologia Avanzada. Madrid: Díaz de Santos, S.A.

Reyes Salgado, M., \& Provedor Fonseca, E. (1998). Factores clínicos epidemiológicos asociados a intoxicaciones agudas por Arsénico en comunidades de San Isidro, Matagalpa. 1998. Tesis de Maestría, Managua.

Rodríguez Barranco, M., Lacasaña, M., Aguilar Garduño, C., Alguacil , J., Gil, F., González Alzaga, B., et al. (2013). Association of arsenic, cadmium and manganese exposure with neurodevelopment and behavioural disorders in children: a systematic review and meta-analysis. Science of the total.

Smith, A. H., Hopenhayn - Rich, C., Warner, M., Biggs, M. L., Moore, L., \& Smith, M. T. (1993). Rationale for selecting exfoliated bladder cell micronuclei as potential 
biomarkers for arsenic genotoxicity. J.

Toxicol. Environ. Health, 40, 223-234.1993

Stynze Ramírez, J. (En Ejecución). Evaluación

de la Contaminación por Arsénico en las Aguas Subterráneas de las Comunidades Tecuaname y El Papalonal, ubicadas en el Municipio de La Paz Centro, Departamento de León, 2016. Tesis de Maestría, Managua.

Wickre, J., Folt, C., Sturup, S., \& Karagas, M. (2004). Environmental Exposure and Fingernail Analysis of Arsenic and Mercury in Children and Adults in a Nicaraguan Gold Mining Community. Archives of Environmental Health, 59(8), 400-409. 